\title{
Influence of the Structure of the Sterol Molecule on Sterol-induced Reproduction in Phytophthora cactorum
}

\author{
C. G. ELLIOTT \\ Botany Department, University of Glasgow, Glasgow $G 128 Q Q$
}

(Received 17 January 1979)

Production of oospores by Phytophthora cactorum in media supplemented with ten $\Delta^{5}$-sterols, differing in the structure of their side chains, has been measured. Spore production increased with increasing size of the substituent at C-24: $\mathrm{H}<\mathrm{CH}_{3}<\mathrm{C}_{2} \mathrm{H}_{5}$. A $24 \alpha$-methyl group conferred greater activity than a $24 \beta$-methyl, but there was less difference between $\Delta^{5}$-sterols with $24 \alpha$ - and $24 \beta$-ethyl groups. There was a greater difference between $\Delta^{7}$-sterols with $24 \alpha$ - and $24 \beta$-ethyl substituents. A double bond at C-22 had no effect on spore production when trans, but reduced activity when cis.

Four $\Delta^{7}$-sterols have been compared with their corresponding $\Delta^{5}$-sterols. Fewer oospores were produced at low concentrations $\left(0.2\right.$ to $\left.1 \mathrm{mg} \mathrm{l}^{-1}\right)$ of each $\Delta^{7}$-sterol than with the $\Delta^{5}$-sterol, but at higher concentrations $\left(10\right.$ to $30 \mathrm{mg} \mathrm{l}^{-1}$ ), the numbers with the $\Delta^{7}$-sterol bore no consistent relation to the numbers with the $\Delta^{5}$-sterol.

The difference between two sterols in promoting sexual reproduction in Phytophthora cactorum is not apparently related to differences in uptake into the mycelium, or to known effects on properties of membranes, but is believed to be due to differences in metabolism of the sterols, and to differences in the hormone activity of compounds derived from them.

\section{INTRODUCTION}

The fungus Phytophthora cactorum is unable to synthesize sterols. It can grow on a synthetic medium without sterols, but such cultures remain purely vegetative. Addition of sterols increases vegetative growth and leads to the onset of sexual reproduction (Hendrix, 1970; Elliott, 1977). Sterols differ in their ability to promote reproduction as measured by the numbers of oospores produced. Cultures supplemented with the $\mathrm{C}_{29}$ sterols, sitosterol, stigmasterol, fucosterol and avenasterol, produce more spores than those supplemented with the $\mathrm{C}_{27}$ sterols, cholesterol and 7-dehydrocholesterol. Cholestanol induces formation of oogonia, but few of them mature into oospores (Elliott, 1972).

This paper reports a more extensive study of oospore production in $P$. cactorum as influenced by various features of sterol structure, namely the size of the substituent at C-24 of the side chain and its orientation, the double bond at C-22 in the side chain, and the position of double bonds in ring $B$. The effect of a particular structural feature was assessed by comparing oospore production with two sterols which differed in respect of this one feature only; the pairs of compounds compared are indicated in Table 3. The structures of the sterols and their systematic names are given in Fig. 1.

\section{METHODS}

The medium, cultural conditions etc., and the method of spore counting, were as described previously (Elliott et al., 1966; Elliott, 1968, 1972). Oospores were produced in cultures in Petri dishes (5 cm diam.) containing medium supplemented from serial dilutions of diethyl ether solutions of the pure sterol alcohols 


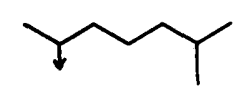

I

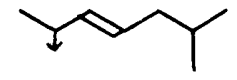

II

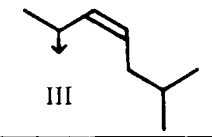

$24 \beta$

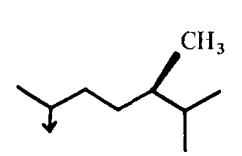

IV
$24 \alpha$

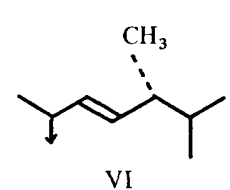

VI

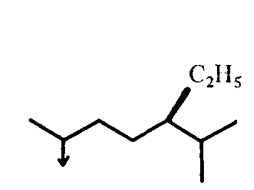

VII<smiles>CC(C)/C=C/C(C(C)C)C(C)(Cl)Cl</smiles>

VIII

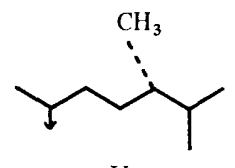

V<smiles>CC(C)CCC(C)C(C)C</smiles>

IX<smiles>[Y]C(C)C(C)C(C)/C=C/C(C)C</smiles>

$\mathrm{X}$

R2

$\Delta^{7}$-Cholestenol [5 $\alpha$-cholest-7-en-3 $\beta$-ol]

Cholesterol [5-cholesten- $3 \beta$-ol]

trans-22-Dehydrocholesterol

[5,22E-cholestadien-3 $\beta$-ol]

III $\quad c i s-22-D e h y d r o c h o l e s t e r o l$

$[5,22 Z$-cholestadien- $3 \beta$-ol]

IV Campesterol [(24R)-24-methyl-5-

cholesten-3 $\beta$-ol]

V $\quad \Delta^{5}$-Ergostenol [(24S)-24-methyl-5-

cholesten-3 $\beta$-ol]

VI Brassicasterol [(24R)-24-methyl-5,22Echolestadien-3 $\beta$-ol]

VII Sitosterol [(24R)-24-ethyl-5-cholesten$3 \beta$-ol]

VIII Stigmasterol [(24S)-24-ethyl-5,22Echolestadien-3 $\beta$-ol]

IX Clionasterol [(24S)-24-ethyl-5cholesten-3 $\beta$-ol]

$\mathrm{X} \quad$ Poriferasterol [(24R)-24-ethyl-5,22Echolestadien-3 $\beta$-ol]<smiles>CC12CCC3C(=CCC4CC(O)CCC43C)C1CCC2O</smiles>

$\mathrm{R} 2$

Fig. 1. Structures and names of sterols used in this work.

(expts 1 to 7) or their acetates (expts 8 to 15). Medium was dispensed in $25 \mathrm{ml}$ volumes, from which five dishes were poured. In experiments 1 to 5 , spore production at five sterol concentrations differing by a factor of $2 / 5$ was studied. In experiments 6 to 15 , eight sterol concentrations differing by a factor of $1 / 2$ were used.

In experiments 1 to 6 , two $25 \mathrm{ml}$ volumes of medium were supplemented with the same concentration of 
sterol, and these provided separate estimates of oospore production based on 16 counts (generally four counts in each of four Petri dishes). In experiments 7 to 15 , only one estimate of spore production was obtained for each sterol concentration, based on 20 counts (generally four counts in each of five Petri dishes, but sometimes five counts in each of four dishes). Sterols were compared by the parameters of the relationship between the number of oospores produced and sterol concentration.

Most of the sterols used were gifts from Dr H. W. Kircher, who prepared them from authentic starting materials using rational, stereospecific synthesis, and who assessed their purity by melting point, mixed melting point and spectroscopic properties (especially nuclear magnetic resonance). Where possible, gasliquid chromatographic analysis confirmed the purity as being at least $99 \%$ in all cases.

\section{RESULTS}

The mean numbers of oospores counted in experiments 1 and 4 are shown in Table 1 and those in experiments $6,7,12,13,14$ and 15 in Table 2 . Account is also taken of results from experiments $2,3,8$ and 9 but these are not shown in detail.

\section{Relation of oospore production to sterol concentration: statistical analysis}

There is no a priori model for the relationship between the number of oospores produced and sterol concentration, but it was found empirically that in most cases the square root of the mean spore number $y$ was linearly related to the logarithm of the sterol concentration $x: \sqrt{y}=a+b \log x$.

The use of the square root of spore number can be justified on several grounds. (1) The ideal sample would be a sector of the Petri dish, but in fact spores were counted in a radial strip of constant width (i.e. the width of the microscope field: $0.72 \mathrm{~mm}$ in expts 1 to 13 and $1.05 \mathrm{~mm}$ in expts 14 and 15). The higher sterol concentrations were characterized by an increased proportion of spores being towards the centre of the colony while at lower concentrations spores were confined to its edge. Hence using the square root corrects against the bias in favour of the inner parts of the colony. (2) Where a good fit could be obtained to both the models $\sqrt{ } y=a+b \log x$ and $y=a^{\prime}+b^{\prime} \log x$ (e.g. expt 6), a larger proportion of the variation between sterol concentrations could be accounted for by the linear regression component in the former case. (3) While in many cases the estimates, $y_{i 1}$ and $y_{i 2}$, of spore production at sterol concentration $x_{i}$ were significantly different, $\left(\sqrt{ } y_{i 1}-\sqrt{ } y_{i 2}\right)^{2}$ was not correlated with $\left(\sqrt{ } y_{i 1}+\sqrt{ } y_{i 2}\right)$, and thus the regression of $\sqrt{ } y$ on $\log x$ could be calculated without weighting. In experiments 1 and 2 but not $6,\left(y_{i 1}-y_{i 2}\right)^{2}$ increased as $\left(y_{i 1}+y_{i 2}\right)$ increased.

In experiments 1 to 3 and 6 , the variation in $\sqrt{ } y$ about the regression line was not significantly different from that between replicate counts, so that the linear model was fully justified. However, in experiment 4 , the variation in $\sqrt{ } y$ about the regression line was significantly greater than that for replicates. In experiments 7 to 15 there were no independent estimates of spore production at each concentration. Therefore, to test the adequacy of the linear model, the 20 counts were divided into two lots of 10 (two dishes each, with the counts of the fifth dish split between them) and the residual variation between concentrations was tested against the variation between those sub-samples. In experiment 7 , the linear model was found valid for stigmasterol and brassicasterol but not for $\Delta^{5}$-ergostenol and sitosterol; in experiment 15, it was not found adequate for campesterol, sitosterol or stigmasterol.

The failure of the linear model in experiments 4 and 15 (campesterol and sitosterol) was due to the oospore number not continuing to increase at the highest sterol concentrations. Rather, it appeared to reach a limiting value; with cholesterol, especially in experiments 4 and 12, the oospore number actually decreased at the highest concentrations tested (Tables 1 and 2). A model which allows for a limiting value is $\sqrt{ } y=\sqrt{ } y_{\max }\left(1-\mathrm{e}^{-k x}\right)$, which can be represented in linear transformation by the equation $1 / \sqrt{ } y=c+d / x$. This model in its linearized form was also fitted. A weighted regression was used, the weighting factors being $y_{i}{ }^{3} / s_{i}^{2}$ where $s_{i}$ is the estimated standard error of $y_{i}$. Comparison of sterols by the parameters $c$ and $d$ on this model led on the whole to the same conclusions as reached using $a$ and $b$ on 


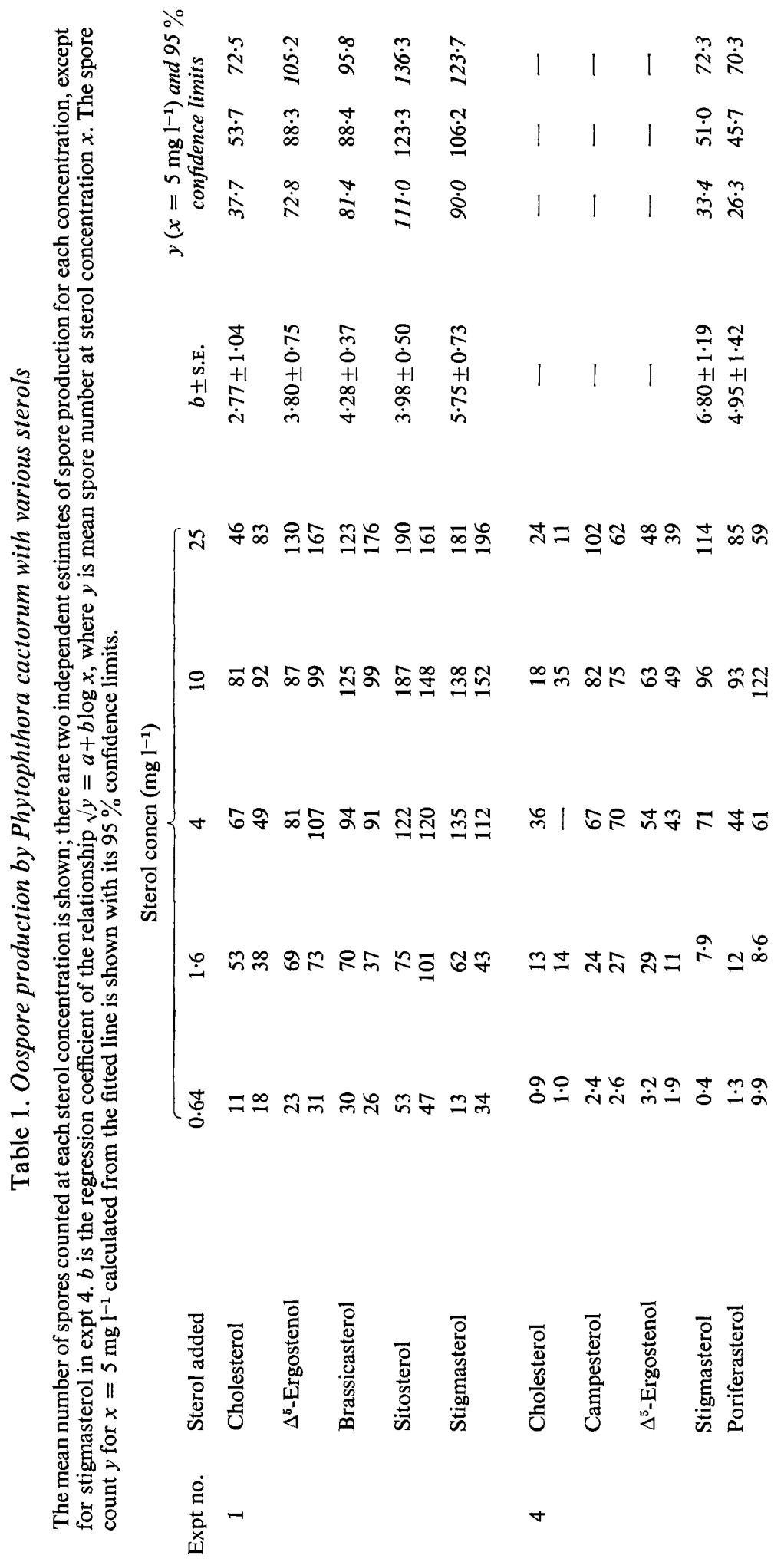




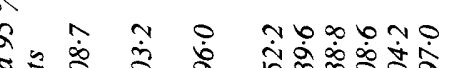

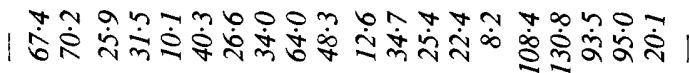

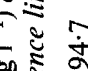

ま்

$\stackrel{\circ}{\dot{\sigma}} \stackrel{\infty}{\dot{\infty}}$

$\ddot{\sim} \dot{\sim} \dot{\sim} \dot{0} \dot{q} \dot{0}$

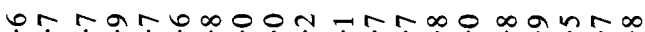

¿

$\dot{\infty} \stackrel{n}{\infty}$

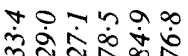

斿

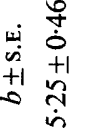

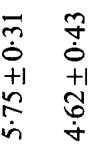

वे

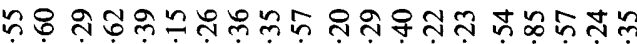

0ं் $\dot{0} \dot{0} \dot{0}$

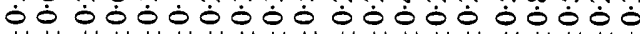

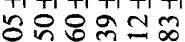

$+1+1+1+1+1+1+1+1+1+1+1+1+1+1+1+1+1+1+1+1$

$\dot{m} \dot{m} \dot{m} \dot{v} \ddot{b} \dot{m}$

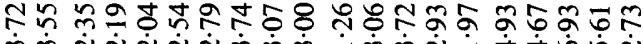

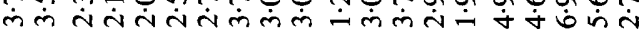

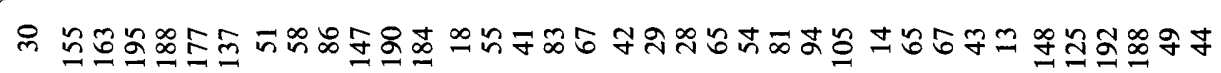

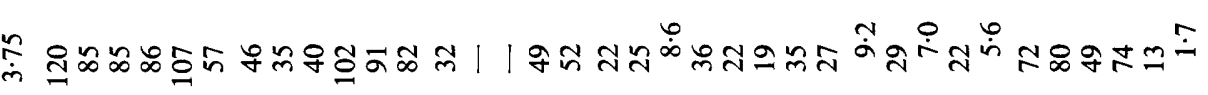

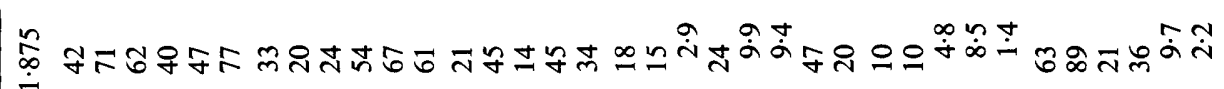

向

守

芛

$\stackrel{*}{2}$

$\stackrel{*}{ \pm}$

$\stackrel{*}{\sim}$ 
Table 3. Tests of significance of the difference in parameters defining oospore-producing activity for various pairs of sterols

The values given are for $t$ testing the differences in $b$ and the calculated value of $\sqrt{ } y$ for the relationship $\sqrt{ } y=a+b \log x$, where $y$ is the mean spore number at sterol concentration $x$. There are 6 degrees of freedom for expts 1 to 5 and 12 for expts 6 to 15 , except where indicated.

Sterols compared

Differences in size and orientation of $\mathrm{C}-24$ substituent

Cholesterol/ $\Delta^{5}$-Ergostenol

Campesterol/ $\Delta^{5}$-Ergostenol

Campesterol/Sitosterol

Sitosterol/Clionasterol

Stigmasterol/Poriferasterol

Differences in absence/presence of $\Delta^{22}$

$\Delta^{5}$-Ergostenol/Brassicasterol

Sitosterol/Stigmasterol

Cholesterol/trans-22-Dehydrocholesterol

Cholesterol/cis-22-Dehydrocholesterol

Difference in $\Delta^{7}$ compared with $\Delta^{5}$

Cholesterol $/ \Delta^{7}$-Cholestenol

$\Delta^{5}$-Ergostenol $/ \Delta^{7}$-Ergostenol

Campesterol/ $\Delta^{7}$-Campestenol

Sitosterol $/ \Delta^{7}$-Stigmastenol

Stigmasterol/ $\alpha$-Spinasterol
Expt no.

4

$12 \dagger$

$13 \dagger$

$14 \uparrow$

4

$12 \dagger$

$13 \dagger$

$14 \uparrow$

$13 \dagger$

$15 \dagger$

$12 \dagger$

$13 \dagger$

$15 \dagger$

4

6

1

7

$13 \dagger$

1

6

$7 \ddagger$

$9 \dagger$

$15 \dagger$

$13 \uparrow$

$13 \dagger$

3

7

$14 \dagger$

$14 \dagger$

3

$7 \ddagger$

$9 \dagger$

$15 \dagger$ $b$

0.777

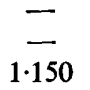

$5 \cdot 618^{* *}$

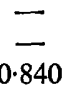

0.840

0.352

$\overline{1 \cdot 406}$

0.263

0.203

$1 \cdot 109$

1.067

0.992

$2 \cdot 136$

0.826

0.919

2.141

1.991

0.916

2.236*

$1 \cdot 726$

2.209*

0.230

0.621

2.907*

0.783

3.049*

1.356

3.158*

$2 \cdot 760$ *

1.808

6.256**

$$
\sqrt{ } y\left(x=5 \mathrm{mg} \mathrm{l}^{-1}\right)
$$

0.735

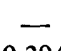

0.394

$5 \cdot 257^{* *}$

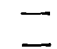

$6 \cdot 187^{* *}$

3. $772^{* *}$

-

4.810**

0.805

0.432

2.773*

0.894

1.028

0.889

0.472

1.763

$1 \cdot 570$

1.609

0.085

0.743

$1 \cdot 121$

1.665

0.038

$5 \cdot 717 *$

0.330

1.551

$8 \cdot 133^{* *}$

2.829*

$1 \cdot 868$

$2 \cdot 427$ *

1.019

9.615**

* Significant at $5 \%$ level. ${ }^{* *}$ Significant at $1 \%$ level.

$\dagger$ Sterols supplied as acetates.

$\ddagger 11$ degrees of freedom.

the first model, and neither model had any evident advantages over the other. Only the results of fitting the first model, $\sqrt{ } y=a+b \log x$, are given. Estimates of the regression coefficient $b$ and the expected value of $\sqrt{ } y$ for $x=5 \mathrm{mg} \mathrm{l}^{-1}$ were obtained with their standard errors and the $95 \%$ confidence interval of $y$ for $x=5 \mathrm{mg} \mathrm{l}^{-1}$ (Tables 1 and 2). These quantities were used to test for differences in activity of the sterol pairs in each experiment (Table 3). In those cases (e.g. expt 15) where the model is not strictly valid, it is considered a sufficiently good approximation to estimate the parameters and use them with due caution. 


\section{Comparison of sterols}

Size and orientation of $\mathrm{C}-24$ substituent. Activity in promoting oospore formation increased with the size of the $\mathrm{C}-24$ substituent, $\mathrm{H}<\mathrm{CH}_{3}<\mathrm{C}_{2} \mathrm{H}_{5}$, but methyl substituents with $\alpha$ orientation had greater activity than those $\beta$-orientated, so that $\Delta^{5}$-ergostenol had an activity hardly different from that of cholesterol, but campesterol was much more active. For cholesterol and $\Delta^{5}$-ergostenol in experiments 1 and 13, neither the regression coefficients nor the estimated value of $\sqrt{y}$ for $x=5 \mathrm{mg} \mathrm{l}^{-1}$ differed significantly. In experiment 14 , on the other hand, the spore number with $\Delta^{5}$-ergostenol increased more rapidly with increasing concentration, and the expected number at $5 \mathrm{mg} \mathrm{l}^{-1}$ was significantly higher. In experiments 4 and 12, where the linear model was not applicable and the statistics were not obtained, the oospore numbers were somewhat higher for $\Delta^{5}$-ergostenol, at least at the higher sterol concentrations. For $\Delta^{5}$-ergostenol and campesterol, in experiments 13 and 14 the regression coefficients did not differ significantly but the estimated value of $\sqrt{y}$ for $x=5 \mathrm{mg} \mathrm{1}^{-1}$ was greater for campesterol; the lines for these two compounds were parallel, with the numbers for campesterol being greater at all concentrations. The results for experiments 4 and 12, though not testable on the model, are in agreement. The activity of campesterol was thus also greater than that of cholesterol.

Sitosterol appeared somewhat more active than campesterol. In experiment 13 , the regression coefficients were not significantly different, but the estimated oospore numbers for $5 \mathrm{mg} \mathrm{l}^{-1}$ were significantly greater for sitosterol. The numbers in experiment 12 support this conclusion, but in experiment 15 there was no difference in activity between the two compounds.

The effect of the orientation of 24-ethyl substituents was examined for the pairs sitosterol/ clionasterol and stigmasterol/poriferasterol. There was no significant difference between stigmasterol and poriferasterol in experiments 4 and 6, or between sitosterol and clionasterol in experiments 12 and 15 . In experiment 13 the estimated oospore number for $5 \mathrm{mg} \mathrm{l}^{-1}$ was lower for clionasterol than for sitosterol, but this was associated with an unusually large difference between the numbers for 30 and $15 \mathrm{mg} \mathrm{l}^{-1}$. The orientation of ethyl groups at C-24 appears to be of much less importance than that of methyl groups in $\Delta^{5}$-sterols. On the other hand, of the two $\Delta^{7,22}$-sterols, $\alpha$-spinasterol and chondrillasterol, the former, with its 24 -ethyl group $\alpha$-orientated, had a higher activity than the latter which has a $24 \beta$-ethyl group (expts 8,15 ). $\Delta^{7}$-Ergostenol also had much lower activity than $\Delta^{7}$-campestenol (expts 8, 14).

Effect of a double bond at C-22. $\Delta^{5}$-Ergostenol and brassicasterol, and sitosterol and stigmasterol, were each compared in several experiments and no differences were detected, except that in experiments 7 and 15 (two out of five comparisons) the slope of the regression line was steeper for stigmasterol than for sitosterol. In experiment 13, the effect of a double bond in the side chain of cholesterol was examined. A trans double bound (as in brassicasterol and stigmasterol) produced no difference in oospore number, but the activity of cis-22dehydrocholesterol was much lower than that of cholesterol.

Effect of a double bond at C-7. With the pairs of sterols compared above, differences in the regression coefficient $b$ were exceptional; differences in activity when present were manifest by differences in the value of $y$ for $x=5 \mathrm{mg} \mathrm{l}^{-1}$. When $\Delta^{7}$-sterols were compared with their $\Delta^{5}$ counterparts, differences in both regression coefficient and in the estimated values of $y$ for $x=5 \mathrm{mg} \mathrm{l}^{-1}$ were found frequently. In some cases the numbers for the $\Delta^{7}$-sterol at high concentrations were similar to (e.g. $\Delta^{7}$-campestenol and campesterol, expt 14; $\Delta^{7}$-cholestenol and cholesterol, expt 3) or even exceeded (e.g. $\Delta^{7}$-stigmastenol and sitosterol, expt 7) those for the $\Delta^{5}$-sterol, but the numbers fell off much more rapidly with decreasing sterol concentration in the case of the $\Delta^{7}$-sterol. In other cases $\left(\Delta^{\mathbf{7}}\right.$ - and $\Delta^{\mathbf{5}}$ ergostenol, expt 14; $\alpha$-spinasterol and stigmasterol, expt 15), fewer oospores were produced with the $\Delta^{7}$-sterol at all concentrations. 


\section{DISCUSSION}

Sterols could differ in their ability to promote oospore formation for a number of reasons. First, there could be differences in uptake and consequently in the sterol content of the mycelium. When cholesterol, campesterol and sitosterol were added to partially grown cultures of $P$. cactorum, their rates of uptake appeared to be similar. When the fungus was fed a mixture of sitosterol and cholesterol, the initial rate of uptake of the sitosterol was greater; however, virtually all the sterol was removed from the medium in about $48 \mathrm{~h}$ (C. G. Elliott \& B. A. Knights, unpublished). Langcake (1975) observed that $P$. infestans took up less sitosterol and stigmasterol than cholesterol and cholestanol. The greater activity of the $\mathrm{C}_{28}$ and $\mathrm{C}_{29}$ sterols therefore cannot be a consequence of the mycelium containing more of them. Sterol esters are taken up more slowly than free sterol alcohols, the esters apparently requiring prior hydrolysis to the alcohol, but with time the uptake of the ester is virtually complete (Elliott \& Knights, 1974). Moreover, when the sterols were supplied as acetates (expts 8 to 15) they showed the same relative activities as when supplied as free sterols (expts 1 to 7). Sterol acetates are deacetylated by the mycelium. Judged by the amounts of sterol recovered from the mycelium (rather than by the amounts left in the medium), $\Delta^{7}$ sterols are initially taken up by the fungus more slowly than $\Delta^{5}$-sterols, but later the uptake of $\Delta^{7}$-sterol approaches that of the $\Delta^{5}$-sterol (Knights \& Elliott, 1976). These analytical studies were all relatively short-term experiments. In the present experiments oospores were counted in mature cultures, and over the period they take to develop it seems unlikely that any differences in initial uptake can be of much importance in determining the differences in oospore-promoting activity.

Modification of the sterol molecule by the fungus could affect its activity. Knights \& Elliott (1976) have shown that whereas $\Delta^{5}$ - and $\Delta^{5,22}$-sterols supplied to $P$. cactorum are recovered from the mycelium unchanged (apart from esterification and production of a small amount of polar material), $\Delta^{7}$ - and $\Delta^{5,7}$-sterols are metabolized to the corresponding $\Delta^{5}$-sterols. Mycelium supplied with $\Delta^{7}$-sterol contains both $\Delta^{7}$ - and $\Delta^{5}$-sterol. The present results show that $\Delta^{7}$-sterols are inferior to the corresponding $\Delta^{5}$-sterols in promoting oospore formation, at least at low concentration. A hypothesis which would explain this observation is that reproduction requires the presence of $\Delta^{5}$-sterols, and that at low concentrations the amount of $\Delta^{5}$-sterol produced from $\Delta^{7}$-sterols which is available for controlling oospore formation is less than when the fungus is supplied with $\Delta^{5}$-sterol.

It has been found (C. G. Elliott \& B. A. Knights, unpublished) that when a mixture of cholesterol and sitosterol is fed to $P$. cactorum the cholesterol is selectively incorporated into the free sterol fraction and the sitosterol into esters and polar metabolites. When supplied singly, there does not appear to be so great a difference in the metabolism of the two sterols, but the results with the mixture may reflect a greater reactivity of sitosterol, which could be related to its greater promotion of oospore formation.

The sterols could affect reproduction through their effects on membrane structure. Very little is known of how sterols other than cholesterol affect the properties of phospholipid membranes. Sterols with side chains longer or shorter than that of cholesterol are less effective than cholesterol itself in increasing the degree of order indicated by electron spin resonance (Craig et al., 1978). These authors did not include in their study sterols with branched side chains like sitosterol with its C-24 substituent. However, Butler et al. (1970) found that sitosterol and cholesterol were similarly effective. Bruckdorfer et al. $(1968,1969)$ found that the ratio of sterol to phospholipid in a dispersion was similar with cholesterol and sitosterol, but lower with 7-dehydrocholesterol, ergosterol and stigmasterol. Ergosterol and stigmasterol were less effective in reducing molecular packing in a lecithin monolayer, and in reducing the permeability of liposomes, than cholesterol, cholestanol, $\Delta^{7}$-cholestenol and 7-dehydrocholesterol (Demel et al., 1972a, b; De Kruyff et al., 1973). The structures of ergosterol and stigmasterol differ from those of $\mathrm{C}_{27}$ sterols in several respects, and it is not 
clear which of them was responsible for the effect on the model membrane; however, the double bond at C-22 may be critical. Hossack \& Rose (1976) studied protoplasts of Saccharomyces cerevisiae which had a modified sterol composition. They found that protoplasts containing $\Delta^{22}$-sterols (brassicasterol, stigmasterol) were more stable and less readily ruptured than those with sterols with a saturated side chain (cholesterol, 7-dehydrocholesterol, $\Delta^{7}$-ergostenol, campesterol, sitosterol). Nes et al. (1978) found that brassicasterol could support anaerobic growth of Saccharomyces cerevisiae somewhat better than $\Delta^{5}$-ergostenol. Rottem et al. (1971) found that stigmasterol and ergosterol were less effective than sitosterol and cholesterol in promoting growth of mycoplasmas. In P. infestans, Langcake (1974) observed that sitosterol and stigmasterol differed in their effects on vegetative growth measured as hyphal extension, but not as dry weight.

These properties of sterols as they affect membranes do not seem helpful in explaining the relationship between molecule structure and activity in promoting reproduction in $P$. cactorum, where the lack of effect of trans- $\Delta^{22}$ and the importance of the size and orientation of the C-24 substituent are significant. There is a marked difference in activity of sterols with $24 \alpha$ - and $24 \beta$-methyl substituents and a less marked difference between $24 \alpha-$ and $24 \beta$ ethyl- $\Delta^{5}$-sterols which is in line with the differences in their nuclear magnetic resonance spectra (Rubinstein et al., 1976). Svoboda \& Robbins (1968) noted a similar difference in the metabolism of $24 \alpha$ - and $24 \beta$-substituted sterols by the tobacco hornworm, Manduca sexta. There is a greater difference in the effect of $24 \alpha$ - and $24 \beta$-ethyl- $\Delta^{7}$-sterols; this could be a reflexion of the specificity of the $\Delta^{7} \rightarrow \Delta^{5}$ isomerase.

Reproduction in Achyla is controlled by steroid hormones, and the orientation of the side-chain groups has a profound effect on activity of the male hormone, antheridiol (Barksdale et al., 1974). The similarities in reproductive pattern observed throughout the Oomycetes and characteristic of this class of fungi indicate that they must share a similar control mechanism, and it therefore seems likely that reproduction in Pythium and Phytophthora is also controlled by steroid hormones and these hormones must be derived biosynthetically from the sterols supplied in the medium (Elliott, 1977; Elliott \& Sansome, 1977; McMorris, 1978). The variation in the effectiveness of sterols in promoting reproduction, associated with differences in the side-chain structure, is, I suggest, due to differences in hormonal activity of compounds derived from the sterols added to the medium, or differences in rates of conversion of individual sterols to similarly active hormonal compounds.

I am greatly indebted to $\mathrm{Dr} \mathrm{H}$. W. Kircher who provided most of the sterols used in this work. Dr J. L. Goad and Dr G. W. Patterson provided certain other samples. I wish to thank Dr R. A. Elton and Mr A. D. McLaren for advice on statistical analysis of the data, and Dr B. A. Knights for advice on chemical matters.

\section{REFERENCES}

Barksdale, A. W., McMorris, T. C., Seshadri, R., Aranachalam, T., Edwards, J. A., Sundeen, J. \& Green, D. M. (1974). Response of Achlya ambisexualis E87 to the hormone antheridiol and certain other steroids. Journal of General Microbiology 82, 295-299.

Bruckdorfer, K. R., Graham, J. M. \& Green, C. (1968). The incorporation of steroid molecules into lecithin sols, $\beta$-lipoproteins and cellular membranes. European Journal of Biochemistry 4, 512 518.

Bruckdorfer, K. R., Demel, R. A., De Gier, J. \& VAN DEenen, L. L. M. (1969). The effect of partial replacements of membrane cholesterol by other steroids on the osmotic fragility and glycerol permeability of erythrocytes. Biochimica et biophysica acta 183, 334-345.
Butler, K. W., Smith, I. C. P. \& Schneider, H. (1970). Sterol structure and ordering effects in spin-labelled phospholipid multibilayer structures. Biochimica et biophysica acta 219, 514-517.

Craig, I. F., Boyd, G. S. \& Suckling, K. E. (1978). Optimum interaction of sterol side chains with phosphatidylcholine. Biochimica et biophysica acta 508, 418-421.

De KruyfF, B., De Greef, W. J., Van EyK, R. V. W., Demel, R. A. \& Van Deenen, L. L. M. (1973). The effect of different fatty acid and sterol composition on the erythritol flux through the cell membranes of Acholeplasma laidlawii. Biochimica et biophysica acta 298, 479-499.

Demel, R. A., Bruckdorfer, K. R. \& Van Deenen, L. L. M. (1972a). Structural requirements of 
sterols for the interaction with lecithin at the airwater interface. Biochimica et biophysica acta 255, 311-320.

Demel, R. A., Bruckdorfer, K. R. \& VAN DeEnen, L. L. M. (1972b). The effect of sterol structure on the permeability of liposomes to glucose, glycerol and $\mathrm{Rb}^{+}$. Biochimica et biophysica acta 255, 321-330.

Elliott, C. G. (1968). Competition and synergism between cholesterol and cholestanol in oospore formation in Phytophthora cactorum. Journal of General Microbiology 51, 137-143.

ELLIOTT, C. G. (1972). Sterols and the production of oospores by Phytophthora cactorum. Journal of General Microbiology 72, 321-327.

ElliotT, C. G. (1977). Sterols in fungi: their functions in growth and reproduction. Advances in Microbial Physiology 15, 121-173.

Elliott, C. G. \& KNIGHts, B. A. (1974). Uptake and metabolism of cholesterol and cholesteryl oleate by Phytophthora cactorum. Biochimica et biophysica acta 360, 78-87.

Elliott, C. G. \& Sansome, E. (1977). The influence of sterols on meiosis in Phytophthora cactorum. Journal of General Microbiology 98, 141-145.

Elliott, C. G., Hendrie, M. R. \& Knights, B. A. (1966). The sterol requirement of Phytophthora cactorum. Journal of General Microbiology 42, 425-435.

HeNDRIX, J. W. (1970). Sterols in growth and reproduction of fungi. Annual Review of Phytopathology 8, 111-130.
Hossack, J. A. \& Rose, A. H. (1976). Fragility of plasma membranes in Saccharomyces cerevisiae enriched with different sterols. Journal of Bacteriology 127, 67-75.

KNights, B. A. \& ElliotT, C. G. (1976). Metabolism of $\Delta^{7}$ - and $\Delta^{5,7}$-sterols by Phytophthora cactorum. Biochimica et biophysica acta 441, 341346.

LANGCAKe, P. (1974). Sterols in potato leaves and their effects on growth and sporulation of Phytophthora infestans. Transactions of the British Mycological Society 63, 573-586.

LANGCAKE, P. (1975). Uptake of sterols by Phytophthora infestans, their intracellular distribution and metabolism. Transactions of the British Mycological Society 64, 55-65.

McMorRIS, T. C. (1978). Sex hormones of the aquatic fungus Achlya. Lipids 13, 716-722.

Nes, W. R., Sekula, B. C., Nes, W. D. \& Adler, J. H. (1978). The functional importance of structural features of ergosterol in yeast. Journal of Biological Chemistry 253, 6218-6225.

Rottem, S., PFendt, E. A. \& Hayflick, L. (1971). Sterol requirements of $\mathrm{T}$-strain mycoplasmas. Journal of Bacteriology 105, 323-330.

Rubinstein, I., Goad, J. L., Clague, A. D. H. \& Mulheirn, L. J. (1976). The $200 \mathrm{MHz}$ NMR spectra of phytosterols. Phytochemistry 15, 195200.

Svoboda, J. A. \& Robrins, W. E. (1968). Desmosterol as a common intermediate in the conversion of a number of $\mathrm{C}_{28}$ and $\mathrm{C}_{28}$ plant sterols to cholesterol by the tobacco hornworm. Experientia 24, 1131-1132. 